\title{
A model for the origin of the anomalous and very bright UV lines of Fe II in gaseous condensations of the star $\eta$ Carinae
}

\author{
S. Johansson ${ }^{1}$ and V. S. Letokhov ${ }^{2,1}$ \\ 1 Department of Physics, Lund University, PO Box 118, 22100 Lund, Sweden \\ e-mail: Vladilen.Letokhov@fysik.lu.se \\ 2 Institute of Spectroscopy, Russian Academy of Sciences, Troitsk, Moscow region, 142190, Russia
}

Received 13 March 2001 / Accepted 19 July 2001

\begin{abstract}
We present a qualitative physical model of the origin of two very bright UV lines of Fe II at 2507-2509 A, present in high-resolution spectra of gaseous condensations (blobs) close to the central star of $\eta$ Carinae. The spectra have been obtained with the Hubble Space Telescope (HST). The model is based on a type of Bowen mechanism with selective photoexcitation of Fe II by a broad HLy $\alpha$ profile, generated in the HII region and diffusely transferred to the optically thick HI region. The frequency distribution by large Doppler diffusion into the HI region provides a substantial broadening of $\mathrm{HLy} \alpha$, which makes it possible to selectively photo-excite shortlived $(\approx 1 \mathrm{~ns})$ states at $11.2 \mathrm{eV}$ in Fe II. Within the frame of the present model the intense HLy $\alpha$ radiation induces a depletion of the lower, long-lived $(\approx 1 \mathrm{~ms})$ level of the strong UV lines by photoionization. The subsequent recombination of Fe III explains the appearance of the "forest" of narrow Fe II lines. The possibility of a cyclic process is also discussed, where HLy $\alpha$ induces depletion to bound states, which have fast decays in the far-UV to the lower state of the Bowen pumping channel.
\end{abstract}

Key words. atomic processes - line: formation - radiation mechansims: non-thermal - stars: individual: $\eta$ Carinae

\section{Introduction. The problem}

Spectra in high spectral and spatial resolution have been obtained with the Space Telescope Imaging Spectrograph (STIS) onboard the Hubble Space Telescope (HST) of $\eta$ Carinae and its nebula, where the slit has been positioned across the star and along the direction of the bipolar lobes. Distinct emission line spectra appear at particular locations close to the star, and they are very different from the spectrum of the star itself (Gull et al. 1999). A forest of narrow Fe II lines is one of the puzzles in the understanding of the object (Johansson \& Zethson 1999). At a projected position of about $0.2^{\prime \prime}$ above the star an emission line spectrum with narrow and bright UV lines of Fe II is related to gaseous condensations of matter called the Weigelt blobs (Weigelt \& Ebersberger 1986), which are probably located in a disk perpendicular to the expansion direction of the bipolar lobes. In Fig. 1 we show a part of the STIS spectrum around $2500 \AA$, where the central star is positioned at 0 along the $y$-axis, giving the spatial scale in arcseconds. The blob spectrum (at $0.2^{\prime \prime}$ in Fig. 1) contains two spectacular features at vacuum wavelengths 2507.55 and $2509.10 \AA$, which are among the strongest single features in the whole observed spectrum

Send offprint requests to: S. Johansson, e-mail: sveneric.johansson@fysik.lu.se between 2000-10000 Å. The location and extraordinary nature of these lines first became evident in HST data from the Faint Object Spectrograph (Davidson et al. 1995) and the High Resolution Spectrograph (Davidson et al. 1997). The lines have been identified as transitions from two highly excited $(11.1 \mathrm{eV})$ energy levels of Fe II (Johansson \& Jordan 1984). The appearance of these bright UV lines in $\eta$ Carinae has been explained as line fluorescence generated through photoexcitation by HLy $\alpha$ in a Bowen mechanism (Johansson \& Hamann 1993). The blob spectrum contains a number of Fe II lines from highly excited levels in the near-infrared, which are also explained by fluorescence generated by HLy $\alpha$. The small width of the bright UV lines of Fe II implies such a relatively low temperature of both the $\mathrm{Fe}^{+}$ions and the electrons, that it is not sufficient for collisional excitation of highly excited levels of Fe II. This fact and the extraordinary strength of the two features at $\lambda \lambda 2507,2509$ in the blob spectrum of $\eta$ Car suggest a selective excitation mechanism.

It is necessary to explain the following features of the anomalous UV lines $(\lambda \lambda 2507,2509)$ of Fe II:

(1) The high brightness of the lines within the framework of the photoselective excitation mechanism of Fe II by HLy $\alpha$ proposed by Johansson \& Hamann (1993);

(2) The narrow spectral width $\left(\Delta \nu / \nu<2 \times 10^{-5}\right)$ of these spectral lines (Johansson \& Zethson 1999); 


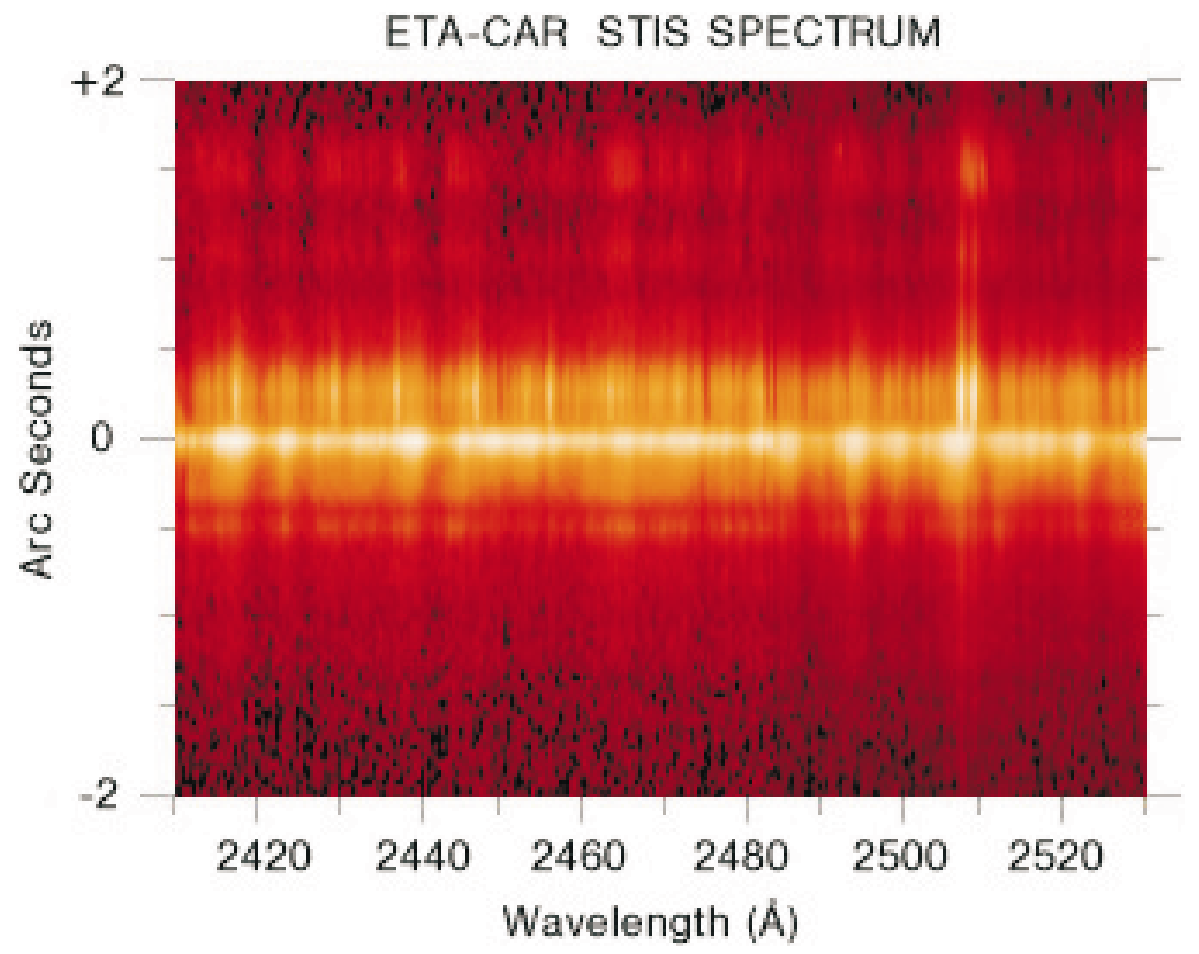

Fig. 1. Extract from the high resolution UV spectrum of the massive star $\eta$ Carinae recorded with the Space Telescope Imaging Spectrograph (STIS) onboard the Hubble Space Telescope. The vertical scale gives the distance (in arcseconds) from the central star (located at 0) to the radiating "blobs" and other gaseous condensations of matter expelled from the star. Of special interest is a blob at about $0.2^{\prime \prime}$ "above" the star, showing a distinct emission line spectrum on a very weak continuous background. Two extraordinary features around 2507.5 and $2509.1 \AA$ (vacuum rest wavelengths) in the blob spectrum require a specific excitation mechanism to be explained.

(3) The anomalous (different from laboratory data) ratio between the intensities of two strong UV lines and their satellite lines, which was the basis for the suggestion of stimulated amplification of these lines (Johansson et al. 1996).

In this paper we consider an excitation mechanism for the $\lambda \lambda 2507,2509$ lines, which is based on a step-wise photoexcitation and photoionization of Fe II by intense HLy $\alpha$ radiation at $1215 \AA$. HLy $\alpha$ is generated in the Weigelt blobs by the absorption of blackbody radiation $(h \nu>$ $13.6 \mathrm{eV}$ ) from the central star and trapped within the optically thick HI/HII media of the blobs. This results in the presence of many recombination lines of Fe II.

\section{Experimental and observational data}

A schematic diagram of the energy levels, radiative transitions and spectroscopic notations of Fe II involved in the discussion of the lines are shown in Fig. 2. The photoselective excitation from the low-lying metastable state $\mathrm{a}^{4} \mathrm{D}_{7 / 2}$ in the radiative transitions $a^{4} \mathrm{D}_{7 / 2}-5 \mathrm{p}^{6} \mathrm{~F}_{9 / 2}, \quad 4 \mathrm{p}^{4} \mathrm{G}_{9 / 2}$ ( $m \rightarrow 2$ allowed transition) produces the intense UV fluorescence of Fe II. The four spectral lines of the radiative transitions from $5 \mathrm{p}^{6} \mathrm{~F}_{9 / 2}$ and $4 \mathrm{p}^{4} \mathrm{G}_{9 / 2}$ to the long-lived states $\mathrm{c}^{4} \mathrm{~F}_{9 / 2}, \mathrm{c}^{4} \mathrm{~F}_{7 / 2}(2 \rightarrow 1$ transitions $)$ consist of 2 strong lines ( $A, C$-lines), which terminate on the $c^{4} \mathrm{~F}_{7 / 2}$ level, and 2 weak lines $(b, d)$, which terminate on the $c^{4} \mathrm{~F}_{9 / 2}$ level.
The four spectral lines have anomalous relative intensities, and the two strongest lines have also anomalous absolute intensities.

According to observational data (Davidson \& Humphreys 1997), the blobs (for example, blob B) are compact condensations (slow-moving compact ejecta) with a $\mathrm{H}$ concentration $N_{\mathrm{H}} \simeq 10^{6} \mathrm{~cm}^{-3}$, and a $\mathrm{Fe}$ concentration $N_{\mathrm{Fe}} \simeq 10^{2} \mathrm{~cm}^{-3}$. The blob diameter is $D \leq 10^{15} \mathrm{~cm}$, and the distance from the central star is $R_{\mathrm{b}} \simeq 3 \times 10^{16} \mathrm{~cm}$. The size of the photosphere of the central star is $d \simeq 3 \times 10^{13} \mathrm{~cm}$, and its effective temperature is $T_{\mathrm{eff}}^{\mathrm{st}} \simeq(20-30) \times 10^{3} \mathrm{~K}$.

These stellar parameters can be evaluated more precisely from future observations. For example, according to recent observations (Gull 2001, private communication) the angular spacing between blob $\mathrm{B}$ and the central star $\sim 0.15^{\prime \prime}$ or $R_{\mathrm{b}} \simeq 3 \times 10^{15} \mathrm{~cm}$, i.e. one order of magnitude closer than obtained from previous data. This new distance is only hundred times larger than the diameter of the central star. However, our model is quite general and can be applied for any blob at any distance from the star.

Extra ultraviolet (EUV) light from the central star with photon energies of $h \nu>13.6 \mathrm{eV}$ converts HI to HII by photoionization. A recombination process $(\mathrm{HII}+\mathrm{e} \rightarrow$ $\left.\mathrm{H}^{*} \mathrm{I}\right)$ provides the intense $\mathrm{HLy} \alpha$ radiation in the optically dense blob medium, where Doppler diffusion causes a broadening of the HLy $\alpha$ emission line from the initial 
(i)

\section{$16,16 \mathrm{eV}$}

\section{Fe III}

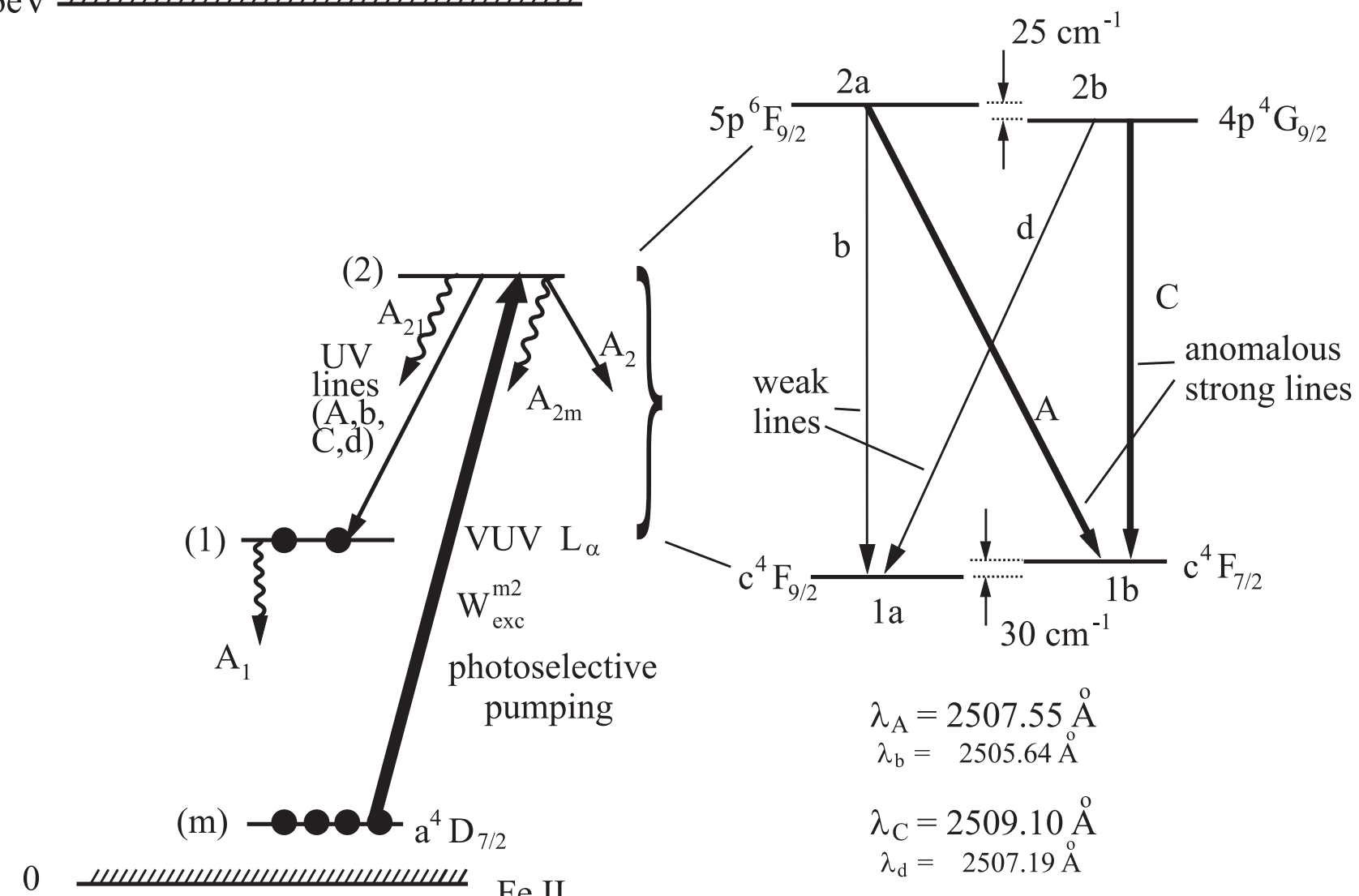

Fig. 2. Energy levels (left side) and quantum transitions (vacuum wavelengths) of Fe II (right side) of relevance to the anomalous UV spectral lines shown in Fig. 1.

Doppler width $\delta \nu_{\mathrm{D}}(\mathrm{H})=6 \mathrm{~cm}^{-1}\left(T_{\mathrm{H}} \simeq 10^{4} \mathrm{~K}\right)$ to a width of up to $320 \mathrm{~cm}^{-1} \gg \delta \nu_{\mathrm{D}}$ (Davidson \& Humphreys 1997).

\section{Concentration of $\mathrm{HI}$ and $\mathrm{HII}$ in the nearest blob of $\eta$ Carinae}

The estimation of the optical density $\tau_{0}$ of HLy $\alpha$ is based on the wavelength detuning of HLy $\alpha$ at $1215.671 \AA$ relative to the wavelengths of two closely spaced spectral transitions in Fe II, namely, $\mathrm{a}^{4} \mathrm{D}_{7 / 2}-4 \mathrm{p}^{4} \mathrm{G}_{9 / 2}(1218.213 \AA)$ and $\mathrm{a}^{4} \mathrm{D}_{7 / 2}-5 \mathrm{p}^{6} \mathrm{~F}_{9 / 2}$ (1217.848 $\AA$ ) (Johansson 1978), which absorb the HLy $\alpha$ radiation. This frequency detuning is $\Delta \nu \cong 160 \mathrm{~cm}^{-1}$. For the long-wavelength wing of the HLy $\alpha$ emission line of neutral hydrogen to coincide with the comparatively narrow absorption lines of $\mathrm{Fe}$ II at about $1218 \AA$ (their internal frequency detuning amounts to some $20 \mathrm{~cm}^{-1}$ ), it is necessary that its optical density is about 1 for a detuning, $\Delta \nu$, relative to the HLy $\alpha$ line center:

$\tau(\Delta \nu)=\sigma(\Delta \nu) N_{\mathrm{HI}} D \simeq 1$,

where $N_{\mathrm{HI}}$ is the concentration of neutral hydrogen, $\sigma(\Delta \nu)$ is the resonance scattering cross-section on the far
$\left(\Delta \nu \gg \delta \nu_{\mathrm{D}}\right)$ Lorentz wing of the natural radiative broadening of HLy $\alpha$. The value of $\sigma(\Delta \nu)$ is determined by a known expression (Mihalas 1978):

$\sigma(\Delta \nu)=\sigma_{0}\left(\frac{\delta \nu_{\mathrm{D}}}{\delta \nu_{\mathrm{rad}}}\right)\left(\frac{\delta \nu_{\mathrm{rad}} / 2}{\Delta \nu}\right)^{2}=\sigma_{0} \frac{\delta \nu_{\mathrm{D}} \delta \nu_{\mathrm{rad}}}{(2 \Delta \nu)^{2}}$

where the first ratio is the Voigt factor, the second one is an effect of detuning, and $\delta \nu_{\mathrm{rad}}=A_{21} / 2 \pi c=2.5 \times$ $10^{-3} \mathrm{~cm}^{-1}$ is the radiative width of HLy $\alpha$. From the Eqs. (1)-(2) we get a simple estimation for the required optical density at the line center of HLya:

$\tau_{0}=\sigma_{0} N_{\mathrm{HI}} D=\frac{\sigma_{0}}{\sigma(\Delta \nu)}=\frac{(2 \Delta \nu)^{2}}{\delta \nu_{\mathrm{rad}} \delta \nu_{\mathrm{D}}} \simeq 7 \times 10^{6}$.

The cross-section $\sigma_{0} \simeq \frac{1}{4} \lambda^{2}\left(\delta \nu_{\mathrm{rad}} / \delta \nu_{\mathrm{D}}\right) \simeq 1.4 \times 10^{-14} \mathrm{~cm}^{2}$, if we insert $\delta \nu_{\mathrm{D}}=1.8 \times 10^{11} \mathrm{~Hz}=6 \mathrm{~cm}^{-1}$ for $T_{\mathrm{H}} \simeq$ $10^{4} \mathrm{~K}$. It means that $N_{\mathrm{HI}} \simeq 5 \times 10^{20} \mathrm{~cm}^{-2}$ in blob B, which is emitting the intense UV lines of Fe II. For the size of blob $\mathrm{B}, D \simeq 10^{15} \mathrm{~cm}$, the required concentration of neutral hydrogen $N_{\mathrm{HI}} \simeq 5 \times 10^{5} \mathrm{~cm}^{-3}$.

For a given value of $N_{\mathrm{HI}}$ it is possible to estimate the value of $N_{\mathrm{HII}}$ on the basis of photoionization/recombination steady-state balance. However, the 
homogeneous photoionization model for the blob is not valid. This conclusion follows from the ratio of the resonant radiative cross-section for Ly $\alpha$ at the center of the line, $\sigma_{0}(\mathrm{Ly} \alpha)$, and the average value of the photoionization cross-section above the ionization limit $\sigma_{\mathrm{ph}}\left(\nu_{\mathrm{c}}\right) \simeq$ $3 \times 10^{-18} \mathrm{~cm}^{2}$ in the spectral range $\left(\nu_{\mathrm{c}}, \nu_{\mathrm{c}}+\Delta \nu_{\mathrm{ph}} \simeq 3 \mathrm{eV}\right)$, where $\Delta \nu_{\mathrm{ph}} \simeq 3 \mathrm{eV}$ is the effective photoionization spectral width in which the Lyman continuum is not drastically reduced. These parameters determine the optical density $\tau_{\mathrm{ph}}$ for the photoionizing radiation in the spectral range $\left(\nu_{\mathrm{c}}, 2 \nu_{\mathrm{c}}\right)$, which provide the main contribution to the photoionization rate $W_{\mathrm{ph}}$ :

$\tau_{\mathrm{ph}} \simeq \frac{\sigma_{\mathrm{ph}}}{\sigma_{0}} \tau_{0}(\mathrm{Ly} \alpha) \simeq 1.4 \times 10^{3} \gg 1$.

The radiation from $\eta$ Car can thus photoionize only a small front part of blob B. This follows from a basic requirement (Eq. (3)) for radiation transfer broadening of Ly $\alpha$, which should excite the absorption line of Fe II. So, the border (Strömgren radius) between the HII zone (the active zone considering the generation of $\mathrm{Ly} \alpha$ ) and the passive HI zone lies inside blob $\mathrm{B}$. The stellar radiation can homogeneously ionize the blob only at frequencies $\nu \geq 10 \nu_{\mathrm{c}}$, where $\tau_{\mathrm{ph}}\left(10 \nu_{\mathrm{c}}\right) \simeq 1$. However, the intensity of the radiation from $\eta$ Car having such an extreme energy is negligible and cannot contribute to the formation of bright UV Fe II lines. This conclusion is valid for the model of the $\eta$ Car photosphere as black body radiation. In case of deviation from the Planck law in the EUV range these estimates have to be modified, of course.

Let us note, that we are in the discussion here and below considering blob $\mathrm{B}$ as a particular example for numerical estimations, as blob B is the strongest emitter of anomalous bright UV Fe II lines. However, the model and exopressions can be used for other stellar parameters of blob $\mathrm{B}$ and for other blobs.

If we assume that the radiation of the central star $\eta$ Car does not suffer from any substantial weakening while traveling the comparatively short distance $R_{\mathrm{b}} \simeq 3 \times 10^{16} \mathrm{~cm}$ (Davidson \& Humphreys 1997) or $3 \times 10^{15} \mathrm{~cm}$ (Gull 2001 , private communication) to the nearest and brightest blob $\mathrm{B}$, then the hydrogen, which is located in the front part of blob B facing $\eta$ Car, should be in its ionized state HII. For this reason, the total hydrogen concentration $N_{0}=N_{\mathrm{HI}}+N_{\mathrm{HII}}$ must be substantially higher than the estimate given above for HI based on the width of $\mathrm{H}$ Ly $\alpha$. A simple estimate for the ratio between the concentrations of HII and HI under steady-state conditions is determined by the ratio between the photoionization rate $W_{\mathrm{ph}}$ of the $\mathrm{HI}$ atom by the radiation of $\eta$ Car with $h \nu>13.6 \mathrm{eV}$ and the recombination rate $W_{\text {rec }}$ of the HII ion:

$\frac{N_{\mathrm{HII}}}{N_{\mathrm{HI}}}=\frac{W_{\mathrm{ph}}}{W_{\mathrm{rec}}} \gg 1$.

The recombination rate of the HII ions depends on their concentration $N_{\mathrm{HII}}$ because the electron concentration $n_{\mathrm{e}}$ in the electrically neutral nebular medium is equal to $N_{\mathrm{HII}}$ :

$W_{\text {rec }}(\mathrm{HII})=\alpha N_{\mathrm{HII}} \quad\left[\mathrm{s}^{-1}\right]$, where

$\alpha=3 \times 10^{-11} \cdot T_{\mathrm{e}}^{1 / 2} \quad\left[\mathrm{~cm}^{3} \mathrm{~s}^{-1}\right]$

and $T_{\mathrm{e}}$ is the electron temperature of the blob. Thus, the concentration of HII is defined by the expression

$N_{\mathrm{HII}}=\left(N_{\mathrm{HI}} \frac{W_{\mathrm{ph}}}{\alpha}\right)^{1 / 2}$.

The photoionization rate $W_{\mathrm{ph}}$ is governed by the flux of photons with $h \nu>13.6 \mathrm{eV}$ from $\eta$ Car, taking into account the dilution factor

$\Omega=\left(\frac{d}{R_{\mathrm{b}}}\right)^{2} \simeq 10^{-6}$,

where $d$ is the diameter of the photosphere of $\eta$ Car and $R_{\mathrm{b}}$ is the distance from blob $\mathrm{B}$ to $\eta$ Car. $W_{\mathrm{ph}}$ also depends on the photoionization cross-section $\sigma_{\mathrm{ph}}(\nu)$ :

$W_{\mathrm{ph}}=\Omega \int_{\nu_{\mathrm{c}}}^{\infty} \sigma_{\mathrm{ph}}(\nu) P(\nu) \mathrm{d} \nu \simeq \sigma_{\mathrm{ph}} I_{\mathrm{ph}}$

where $P(\nu)$ is the spectral brightness (photons $/ \mathrm{cm}^{2} \mathrm{sr} \mathrm{Hz}$ ) of the blackbody radiation from the photosphere of $\eta$ Car, $\sigma_{\mathrm{ph}}$ is an average effective photoionization crosssection introduced above, and $I_{\mathrm{ph}}$ is the integrated intensity (photons $/ \mathrm{cm}^{2} \mathrm{~s}$ ) of the stellar radiation at the blob surface in that spectral range, which gives the main contribution to the photoionization rate. If one describes the radiation spectrum of the photosphere of $\eta$ Car as that of a black body with a temperature of $T=30000 \mathrm{~K}$, one can then approximately estimate the photoionization rate $W_{\mathrm{ph}}$ to be about $0.02-2 \mathrm{~s}^{-1}$ for $R_{\mathrm{b}}=3 \times\left(10^{16}-10^{15}\right) \mathrm{cm}$. For other more remote blobs the value $W_{\mathrm{ph}}$ decreases in proportion to $\left(R_{\mathrm{b}} / R\right)^{2}$, where $R$ is the distance to the other blobs from $\eta$ Car.

\section{The structure of the $\mathrm{HII} / \mathrm{HI}$ region in blob $\mathrm{B}$ of $\eta$ Carinae}

The steady-state concentration of $\mathrm{H}$ atoms in blob B, required in accordance to Eq. (3), is sufficiently high and can completely absorb the ionizing radiation of $\eta$ Car. Indeed, the order of magnitude of the photoionization depth $l_{\mathrm{ph}}$ in blob B may be estimated by the following simple expression:

$l_{\mathrm{ph}} \simeq \delta l_{\mathrm{ph}} \frac{W_{\mathrm{ph}}}{W_{\mathrm{rec}}}=\delta l_{\mathrm{ph}} \frac{N_{\mathrm{HII}}}{N_{\mathrm{HI}}}$,

where $\delta l_{\mathrm{ph}}$ is the thickness of the transition layer between the complete ionization region (HII/HI region) and the region of very weak ionization of HI (by the weak and extremely short-wavelength $(h \nu \gg 13.6 \mathrm{eV})$ radiation of $\eta$ Car), and defined in the same simple approximation as (Mihalas 1978):

$\sigma l_{\mathrm{ph}} \cong \frac{1}{\sigma_{\mathrm{ph}} N_{0}}=\frac{1}{\sigma_{\mathrm{ph}}\left(N_{\mathrm{HI}}+N_{\mathrm{HII}}\right)}$, 
where $\sigma_{\mathrm{ph}}$ is the photoionization cross section above the photoionization limit. For simplicity $\sigma_{\mathrm{ph}}$ is described by some average value of $\sigma_{\mathrm{ph}} \simeq 3 \times 10^{-18} \mathrm{~cm}^{2}$.

We will first estimate the critical hydrogen density $N_{0}^{\text {cr }}$, at which the photoionization depth $l_{\mathrm{ph}}$ satisfies the condition

$l_{\mathrm{ph}} \simeq D$,

which means that the boundary of the Strömgren sphere is exactly at the rearmost layer (opposite to $\eta$ Car) of the blob $\mathrm{B}$, so that the entire volume of the blob is subject to photoionization. We use the Eqs. (8), (10) and (11), considering that the concentration of HII in the HII/HI region suffering photoionization is equal to $N_{0}$, to obtain

$N_{0}^{\mathrm{cr}} \simeq\left(\frac{W_{\mathrm{ph}}^{2}}{\sigma_{\mathrm{ph}} D \alpha}\right)^{1 / 2}=\left(\frac{I_{\mathrm{ph}}}{\alpha D}\right)^{1 / 2}$.

For blob $\mathrm{B}$ we can accept approximately $I_{\mathrm{ph}} \simeq 3 \times$ $10^{16}$ photons $\mathrm{cm}^{-2} \mathrm{~s}^{-1}$, taking into account the dilution factor $\Omega \simeq(0.25-25) \times 10^{-6}$. Correspondingly, from Eq. (14) we get the value $N_{0}^{\text {cr }} \simeq(0.5-5.0) \times 10^{7} \mathrm{~cm}^{-3}$. This means that only at $N_{0}<N_{0}^{\text {cr }}$ the entire volume of blob B is uniformly photoionized, and at $N_{0}>N_{0}^{\mathrm{cr}}$, it is only the foremost layer of the blob with a thickness of $l_{\mathrm{ph}}$ that is photoionized.

As an illustration, Fig. 3 shows the optical density $\tau_{0}$, both in the photoionized region HII/HI and in the HI region, as a function of the total hydrogen concentration $N_{0}$ in the blob under consideration. At $N_{0}<N_{0}^{\mathrm{cr}}=10^{7} \mathrm{~cm}^{-3}$ the entire volume of the blob is uniformly photoionized, and the remaining small proportion of neutral hydrogen atoms provides an optical density $\tau_{0}$ whose value is lower than the necessary value given by Eq. (3). Higher $\tau_{0}$ values can only be reached at $N_{0}>N_{0}^{\text {cr }}$, when only the foremost layer of the blob is photoionized. In the transitional concentration region where $N_{0} \simeq N_{0}^{\mathrm{cr}} \simeq 10^{7} \mathrm{~cm}^{-3}$, the photoionization limit passes inside the volume of the blob, i.e., in the distance range between $R_{\mathrm{b}}$ and $R_{\mathrm{b}}+D$ from $\eta$ Car. In this ionized region, the optical density $\tau_{0}$ reaches its steady-state value, as the reduction of the photoionization volume is being offset by the rise of the recombination rate $W_{\text {rec }}$, which can be seen from the right-hand bottom branch of the curve $\tau_{0}\left(N_{0}\right)$ in Fig. 3. In this HII/HI region, HLy $\alpha$ photons are being born and diffuse isotropically, i.e. into the practically non-ionized HI region as well, where the intensity of the $\operatorname{Ly} \alpha$ radiation is naturally weakened. This HI region has a huge optical density $\tau_{0}$ for the photons, which can reach the required values given by Eq. (3). The optical thickness of the transient layer HII/HI with physical width $\delta l_{\mathrm{ph}}$ can be estimated as:

$\tau_{0}^{\mathrm{tr}} \simeq N_{\mathrm{HI}}^{\mathrm{tr}} \delta l_{\mathrm{ph}} \sigma_{0} \simeq \frac{1}{2} \frac{\sigma_{0}}{\sigma_{\mathrm{ph}}} \simeq 2 \times 10^{3}$

where $N_{\mathrm{HI}}^{\mathrm{tr}} \simeq \frac{1}{2} N_{0}$ is the average concentration of $\mathrm{HI}$ in the transient layer, and $\delta l_{\mathrm{ph}}$ is determined by Eq. (11). The high density of HI compensates the small width of this layer and as a result the optical thickness of the transient layer $\tau_{0}^{\text {tr }}$ is almost equal to the optical thickness $\tau_{0}$ of the whole active zone. Nevertheless, these values of $\tau_{0}$ are much less than the required amount given in Eq. (3) for the Ly $\alpha$ excitation of Fe II. At the same time, the remaining, dissipating (passive) volume of the blob is also large enough to provide for $\tau_{0} \simeq 10^{7}$ (the vertical branch of the curve in Fig. 3) and the appropriate broadening of the Ly $\alpha$ line as a result of the Doppler frequency diffusion of the radiation. The Ly $\alpha$ radiation, diffusing from the active region into this passive volume, can excite the UV fluorescence in Fe II in a photoselective fashion, thanks to the compensation of the detuning between the wavelengths of Ly $\alpha$ and the absorption line of Fe II. The Fe II ions are always being formed in the passive region of the blob on account of photoionization by the radiation of $\eta$ Car in the range $7.6 \mathrm{eV}<h v<13.6 \mathrm{eV}$ that passes through the HII/HI region without suffering practically any absorption through photoionization. Hamann et al. (1999) also noted that the Fe II lines do not form in HII regions, but rather in partially ionized zones behind the HII/HI recombination front.

The above crude qualitative analysis shows that intense UV Fe II lines can be observed only in those blobs having a hydrogen concentration $N_{0}>N_{0}^{\text {cr }}$, which provides for $\tau_{0} \geq 10^{7}$ in the passive HI zone of the blob.

\section{Qualitative discussion of the $\operatorname{Ly} \alpha$ radiation spectrum in the active and passive blob regions}

Based on well-known papers on resonance radiation transfer in optically very thick media (Adams 1972; Harrington 1973; Hummer \& Kunasz 1980), one can qualitatively represent the form of the spectrum of the $\mathrm{Ly} \alpha$ radiation going from the active HII/HI region of the blob into its passive region HI and its further evolution therein. For the sake of brevity, we denote the optical thickness with reference to the center of the Ly $\alpha$ line as $\tau_{0}(\mathrm{HI})=\tau_{0}^{\mathrm{p}}$ in the passive zone and $\tau_{0}(\mathrm{HII} / \mathrm{HI})=\tau_{0}^{\mathrm{a}}$ in the active zone. Figure $4 \mathrm{a}$ shows the spectrum of the recombination line Lya generated in the HII/HI region, its initial Doppler width being $\delta \nu_{\mathrm{D}} \simeq 6 \mathrm{~cm}^{-1}$. The natural line width $\delta \nu_{\text {rad }}$ is narrower by a factor of $10^{3}$ than the Doppler width. Owing to the Doppler frequency diffusion, the line profile of the Ly $\alpha$ radiation gets broadened inside the active region by approximately $\sqrt{\ln \tau_{0}^{a}} \simeq 3.5$ times (Fig. $4 \mathrm{~b}$ ) as a result of a relatively limited number of scattering events. These events shift the photon frequency to the wings of the Doppler profile, where the optical density is low, and the photon being scattered directly escapes from the active region. Accordingly, where scattering is repeated many times as a result of trapping, the intensity of the Ly $\alpha$ radiation is increased $\sqrt{\ln \tau_{0}^{\mathrm{a}}}$ times. The spectrum of the radiation scattered into the surrounding medium features a dip in the center (Fig. 4c), whose total width is $\Delta \nu_{\mathrm{a}} \simeq \delta \nu_{\mathrm{D}} \sqrt{\ln \tau_{0}^{\mathrm{a}}}$. Some of this radiation leaves the blob, the rest of it being scattered (and partially reflected) into the optically 


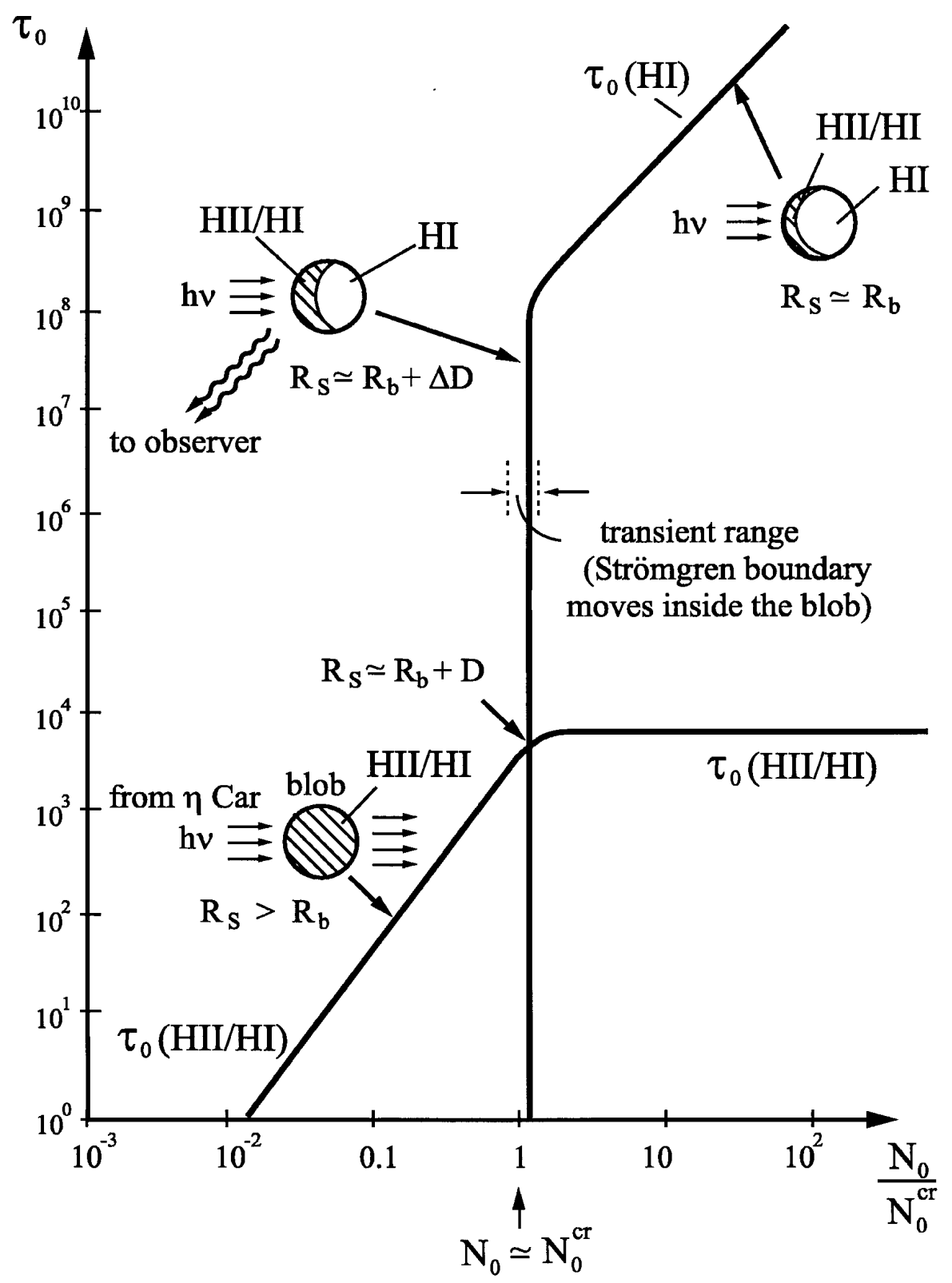

Fig. 3. Optical thickness, $\tau_{0}$, of Ly $\alpha$ (in the center of the line) as a function of the total concentration, $N_{0}$, of neutral (HI) and ionized (HII) hydrogen in blob B. $N_{0}^{\text {cr }}$ is the maximum (critical) density for which the whole volume of blob is ionized. At $N_{0}>N_{0}^{\text {cr }}$ the blob volume is partially ionized, on the front layer of the blob.

denser passive region HI. In the case of multiple scattering in the Lorentzian wing of the Ly $\alpha$ line, the Doppler frequency diffusion broadens the radiation spectrum to a greater value of $\Delta \nu_{\mathrm{p}}$. The red-side Doppler frequency diffusion $\Delta \nu_{\mathrm{p}} / 2$ may become as great as the detuning $\Delta \nu$ of the central frequency of the Ly $\alpha$ radiation from the frequency $\nu_{m 2}$ of the Fe II absorption line (Fig. 4d). With the diffusion being as is, the radiative spectral intensity drops in proportion to the broadening of the spectrum, i.e. by $\left(\Delta \nu_{\mathrm{p}} / \Delta \nu_{\mathrm{a}}\right)$. The contribution to the diffusion from the much narrower Doppler width of Ly $\alpha$ in the passive region is small because the ratio $\delta \nu_{\mathrm{D}} / \Delta \nu_{\mathrm{p}}$ is small.

Let us estimate the width $l_{\mathrm{br}}$ of the border layer adjacent to HII/HI, which provides the required Ly $\alpha$ broaden- ing, i.e. the layer with optical thickness $\tau(\mathrm{HI}) \simeq 7 \times 10^{6}$. The physical thickness of this layer can be estimated in a similar manner as in Eqs. (1)-(3):

$\tau_{0}=\sigma_{0} l_{\mathrm{br}} N_{0} \simeq 7 \times 10^{6}$

where $N_{0}>N_{0}^{\text {cr }}$. For example, in case $N_{0} \simeq 10^{8} \mathrm{~cm}^{-3} \approx$ $10 N_{0}^{\text {cr }}$ the value $l_{\mathrm{br}} \simeq 5 \times 10^{12} \mathrm{~cm}$. This width is much less than the size of blob, but much higher than the width $\delta l_{\text {ph }}$ of the transient zone HII/HI:

$\frac{l_{\mathrm{br}}}{\delta l_{\mathrm{ph}}} \simeq \tau_{0} \frac{\sigma_{\mathrm{ph}}}{\sigma_{0}} \simeq 1.5 \times 10^{3}$ 

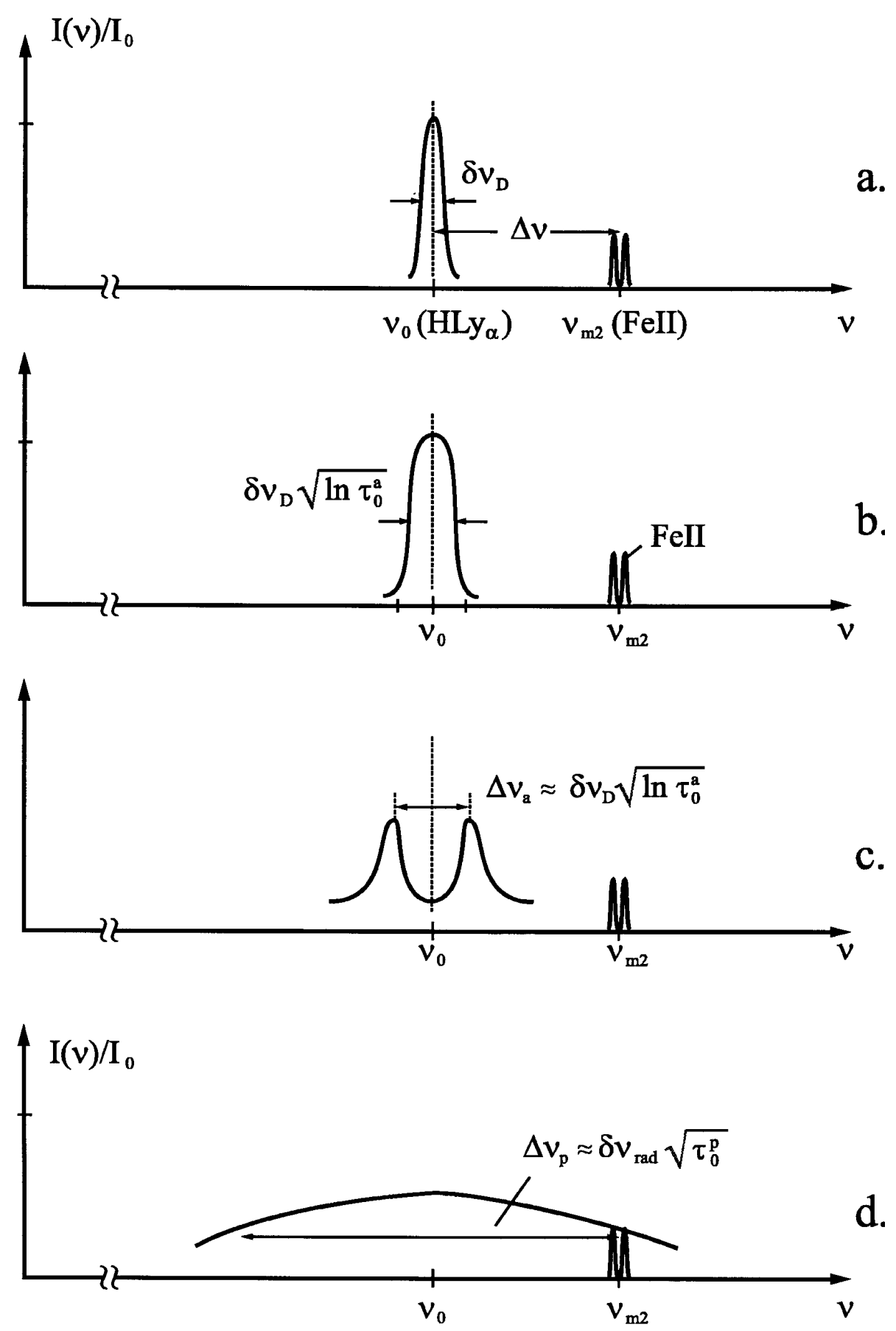

Fig. 4. Line profile of HLy $\alpha$ : a) Doppler-broadened line due to recombination radiation in the HII/HI zone; b) broadening of Ly $\alpha$ due to resonance transfer radiation in the active HII/HI zone; c) spectra of radiation escaping from the active zone; d) broadening of $\operatorname{Ly} \alpha$ due to resonance transfer radiation in the passive HI zone from resonant scattering in the Lorentz wings of natural-broadened spectral line.

The ratio of $l_{\mathrm{br}}$ to the thickness of the active zone is:

$\frac{l_{\mathrm{br}}}{l_{\mathrm{ph}}} \simeq \frac{\tau_{0} N_{0} \alpha}{\sigma_{0}\langle I(\mathrm{Ly} \alpha)\rangle}$.

For $N_{0} \simeq 10 N_{\mathrm{cr}}$, the widths $l_{\mathrm{br}}$ and $l_{\mathrm{ph}}$ of these zones are comparable.

On the basis of such a simple estimation it is possible to imagine a qualitative picture of the spatial sequence of various zones on the leading edge of the blob: (1) photoionized
HII active zone; (2) transient HII/HI zone; (3) line broadening zone; (4) zone of UV Fe II fluorescence as shown in Fig. 5 for idealized homogeneous distribution of hydrogen. Of course, in a more realistic case of a gradual spatial distribution of hydrogen the sharp behavior of the curves takes place in the points (surface) of the Strömgren radius position, which looks as shown in Fig. 3 (upper right corner). 


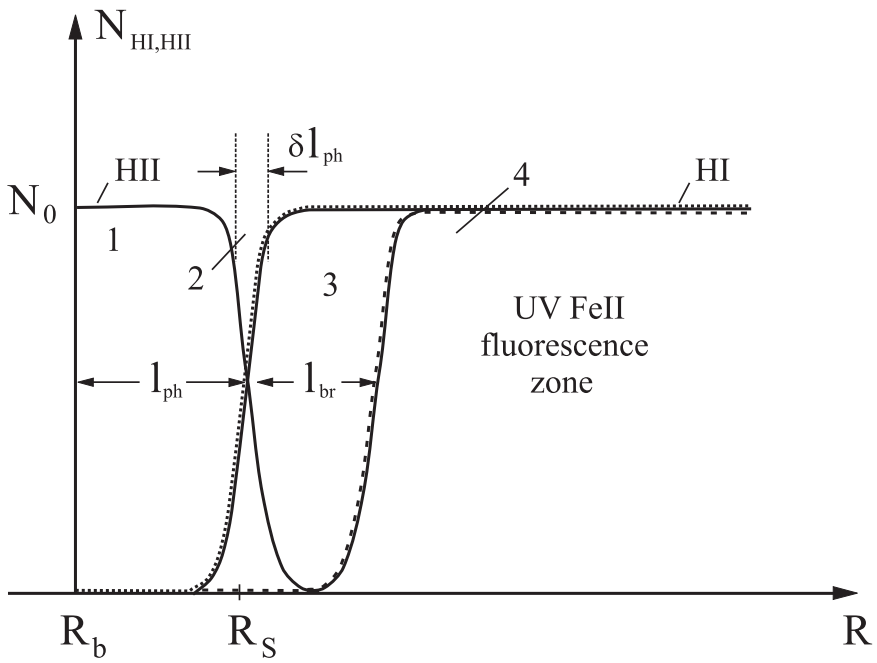

Fig. 5. Spatial distribution of the HII active zone (1), transient HII/HI zone (2) at the distance of the Strø'mgren radius $\left(R_{\mathrm{s}}\right)$, transient HI zone (3) working as a Doppler diffusion broadening medium for the Ly $\alpha$ radiation from the active zone, HI passive zone (4), where the spectrally wide Ly $\alpha$ radiation excites Fe II and forms the UV fluorescence lines.

In all these cases, the region of the blob that emits the UV Fe II lines appears, as observed from the Earth, to approximately occupy the entire volume of the blob.

One should remark, in addition to the above crude estimates, that the passive $\mathrm{HI}$ region is substantially colder than the active, hot region $\mathrm{HII} / \mathrm{HI}$ suffering an intense photoionization by the radiation of $\eta$ Car. The temperature of the HI region largely depends on the photoionization of the elements whose ionization potential $I<$ $13.6 \mathrm{eV}$ and whose concentration is very low in comparison with that of hydrogen, including e.g. Fe I. Regarding the variation of the intensity of the Ly $\alpha$ radiation in the HI region, it can increase because of the longer diffusion pathway of Ly $\alpha$ in the passive region. In our case, however, it impinges upon the boundary between the HII/HI and HI regions. For this reason, the effect of it being scattered back into the active region, where the optical density for the frequencies $\left|\nu-\nu_{0}\right|>\Delta \nu_{\mathrm{a}}$ is low, should be substantial, so that this radiation should escape into the surrounding medium. Moreover, the optical density in the HI region should be additionally increased as a result of the reduction of the Doppler width of $\operatorname{Ly} \alpha$ in the colder passive region.

All these questions require special consideration in the future. What is important now is that the width of the Ly $\alpha$ radiation in the active region of the blob is too small to provide for the resonant excitation of Fe II. One should therefore consider the photoselective excitation of Fe II by the Ly $\alpha$ radiation in the passive region. As the intensity of the Ly $\alpha$ radiation, coming from the active region into the passive one and suffering repeated scattering therein, is indefinite, it is advisable to deal in the subsequent qualitative discussion with the effective temperature of the Ly $\alpha$ radiation. The quantity $T_{\text {eff }}(\operatorname{Ly} \alpha)$ will be used to refer to the radiation intensity of the surface of an equivalent black body with a temperature $T_{\text {eff }}$ in the spectral range $\Delta \nu_{\mathrm{p}}$ corresponding to the spectral width $\Delta \nu_{\mathrm{p}}$ of the Ly $\alpha$ radiation suffering diffusive broadening. Moreover, to consider the entire problem of the origin of the mysterious UV Fe II line we must restrict our consideration to a spatially uniform $T_{\text {eff }}(\operatorname{Ly} \alpha)$ in the fluorescence zone 4 (Fig. 5). The quantitative consideration of the problem is a future task.

\section{Photoselective excitation of Fe II by HLy $\alpha$ in the $\mathrm{HI}$ zone}

To produce bright UV fluorescence lines the rate of photoexcitation of level 2 in Fe II from level $m$ must be substantially higher than the rate of collisional excitation by electrons:

$W_{\mathrm{exc}}^{m 2}(\mathrm{Ly} \alpha) \gg W_{\mathrm{exc}}^{(2)}\left(\mathrm{e}^{-}\right)$.

The rate of resonant excitation of Fe II by HLy $\alpha$ can be estimated by the simple expression

$W_{\mathrm{exc}}^{m 2}(\operatorname{Ly} \alpha)=\sigma_{m 2} P_{\nu, T} \Delta \nu_{m 2} \cdot 4 \pi$,

where $\sigma_{m 2}$ is the cross-section of the radiative transition $m \rightarrow 2, P_{\nu, T}$ is the spectral brightness of HLy $\alpha$ emission with the effective temperature $T=T_{\text {eff }}(\operatorname{Ly} \alpha)$. The crosssection $\sigma_{m 2}$ is given by the expression

$\sigma_{m 2}=\frac{\lambda_{2 m}^{2}}{2 \pi} \frac{A_{2 m}}{\Delta \omega_{m 2}} \frac{g_{2}}{g_{m}} \approx \frac{\lambda_{2 m}^{2}}{2 \pi} \frac{A_{2 m}}{2 \pi \Delta \nu_{m 2}}$,

where $\lambda_{2 m}=1215 \AA$ is the wavelength of the absorption line Fe II $\left(\mathrm{a}^{4} \mathrm{D}_{7 / 2} \rightarrow 5 \mathrm{p}{ }^{6} \mathrm{~F}_{9 / 2}, 4 \mathrm{p}{ }^{4} \mathrm{G}_{9 / 2}\right), A_{2 m} \simeq 10^{8} \mathrm{~s}^{-1}$ is the Einstein coefficient for the $2 \rightarrow m$ radiative decay, and $\Delta \omega_{m 2}=2 \pi \Delta \nu_{m 2}$ is the spectral width of the absorption line $m \rightarrow 2$.

The spectral intensity of HLy $\alpha$ can be presented as black body radiation at $\lambda=\lambda_{m 2}$ with the effective temperature $T_{\text {eff }}$ :

$$
\begin{aligned}
P_{\nu, T} & =\frac{1}{h \nu} B_{\nu, T} \\
& =\frac{2}{\lambda_{2 m}^{2}} \frac{1}{\mathrm{e}^{h \nu_{2 m} / k T_{\text {eff }}}-1}\left[\text { photons } / \mathrm{cm}^{2} \mathrm{sr} \mathrm{Hz}\right]
\end{aligned}
$$

So, according to (20) we get the expression for $\mathrm{W}_{\mathrm{exc}}^{m 2}$ :

$W_{\mathrm{exc}}^{m 2} \simeq \frac{A_{2 m}}{\mathrm{e}^{h \nu_{m 2} / k T_{\text {eff }}}-1}$

Condition (19) will require that the effective temperature of HLy $\alpha$ emission should be

$T_{\mathrm{eff}}(\mathrm{Ly} \alpha)>\frac{h \nu_{2 m}}{k} / \ln \left(1+\frac{A_{2 m}}{W_{\mathrm{exc}}^{(2)}\left(\mathrm{e}^{-}\right)}\right)$.

Note that the requirement in Eq. (24) is independent of both the spectral width $\Delta \nu_{2 m}$ of Fe II and the spectral width of HLy $\alpha$. For illustration and subsequent estimations, Fig. 6 (left-hand scale) shows $\mathrm{W}_{\mathrm{exc}}^{m 2}\left[\mathrm{~s}^{-1}\right]$ as a function of $T_{\text {eff }}(\operatorname{HLy} \alpha)$. 


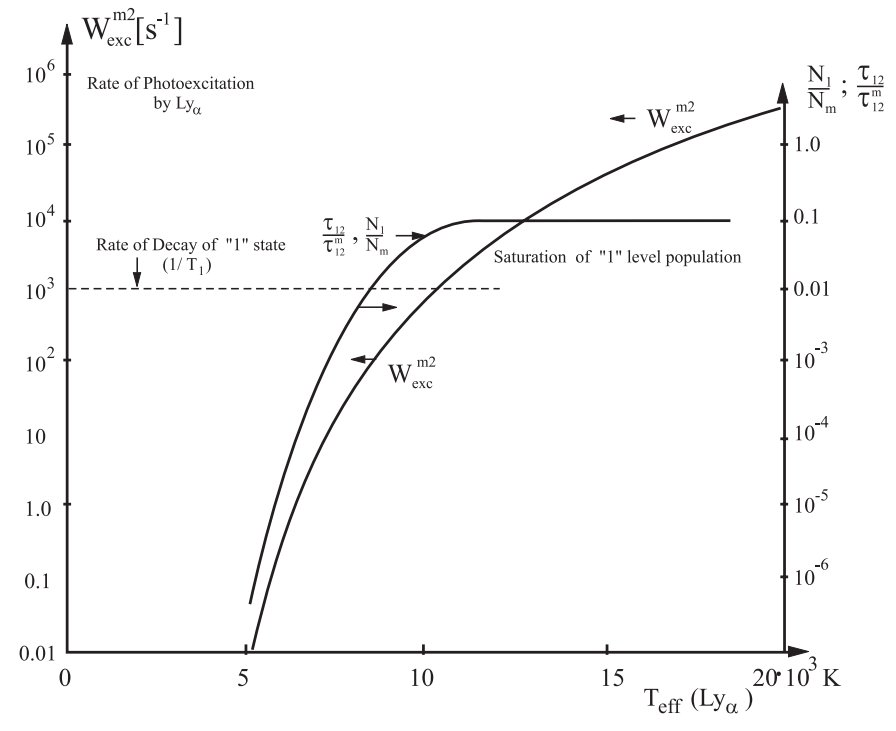

Fig. 6. The rate of photoexcitation of the upper levels $2, W_{\mathrm{exc}}^{m 2}$, the relative population of the lower levels $1, N_{1} / N_{m}$, and the optical density, $\tau_{12}$, of the 1-2 UV transitions of Fe II as functions of the effective temperature of $T_{\mathrm{eff}}(\mathrm{Ly} \alpha)$.

The rate of collisional excitation by electrons with the energy $E$ and mean velocity $v_{\mathrm{e}}$ can be estimated by the expression

$W_{\mathrm{exc}}^{(2)}\left(\mathrm{e}^{-}\right) \simeq n_{\mathrm{e}}<\sigma_{\mathrm{e}} v_{\mathrm{e}}>_{E}$

where $n_{\mathrm{e}}$ is the concentration of electrons comparable with that of the Fe II ions. For the hydrogen concentrations $N_{0} \simeq(1-10) N_{0}^{\text {cr }} \simeq 10^{7}-10^{8} \mathrm{~cm}^{-3}$ the iron concentration would be about $10^{3}-10^{4} \mathrm{~cm}^{-3}$. Due to full photoionization conversion $\mathrm{Fe} \mathrm{I} \rightarrow \mathrm{Fe} \mathrm{II,} \mathrm{the} \mathrm{value} \mathrm{of}$ $n_{\mathrm{e}} \simeq 10^{3}-10^{4} \mathrm{~cm}^{-3}$. Since the average electron energy is in the range $<1 \mathrm{eV}$, there is only a small number of electrons in the energy range $E>E^{*}$, where $E^{*}$ is the energy of the highly excited states $2\left(5 \mathrm{p}^{6} \mathrm{~F}_{9 / 2}\right.$ and $\left.4 \mathrm{p}^{4} \mathrm{G}_{9 / 2}\right)$ of Fe II. Thus, only very few electrons are capable of exciting the high-lying levels 2. An estimate based on the data presented in Shevelko \& Vainstein (1993) shows that $W_{\text {exc }}^{(2)}\left(\mathrm{e}^{-}\right)$is about $10^{-5}-10^{-6} \mathrm{~s}^{-1}$. It follows from the simple estimates above that at $T_{\text {eff }}(\operatorname{Ly} \alpha)>5000 \mathrm{~K}$ (see the left-hand curve $W_{\text {exc }}^{m 2}$ in Fig. 6) the photoselective excitation of levels 2 by the Ly $\alpha$ radiation proves much more effective than collisional excitation by thermal electrons, hence the more intense UV fluorescence of Fe II.

The observed huge intensity of the UV lines of Fe II is due to the high effective temperature of the Ly $\alpha$ radiation that might be about $10^{4} \mathrm{~K}$. With the effective radiation temperature being so high, the photoselective excitation rate $W_{\text {exc }}^{m 2}$ exceeds the relaxation rate of the lower level $1\left(\mathrm{c}^{4} \mathrm{~F}_{7 / 2,9 / 2}\right)$, where the Fe II atoms are accumulated as a result of their fast UV radiative decay (see Figs. 2 and 6). As a consequence of the accumulation of Fe II in these long-lived states, one can expect a substantial optical density for the anomalous UV transitions of Fe II being studied.
The long lifetime of $c^{4} \mathrm{~F}_{7 / 2,9 / 2}$ allows Fe II to be accumulated in these states, and the population of the lower state 1 relative to that of the metastable state $m$ is given by

$\frac{N_{1}}{N_{m}}=\frac{W_{\mathrm{exc}}^{m 2}}{W_{\mathrm{exc}}^{m 2}+\left(1 / T_{1}\right)}$,

where the photoexcitation rate $W_{\text {exc }}^{m 2}$ is defined by Eq. (18) and $T_{1} \approx 1.5 \mathrm{~ms}$ (Kurucz 1988). Expression (26) is valid in the absence of the saturation of the $m \rightarrow 2$ transition, i.e. for $W_{\text {exc }}^{m 2} \ll A_{2 m} \approx 10^{8} \mathrm{~s}^{-1}$ or $N_{2} \ll N_{1}, N_{m}$. The right-hand part of Fig. 6 shows $N_{1} / N_{2}$ as a function of the effective temperature $T_{\text {eff }}(\mathrm{Ly} \alpha)$. At an excitation rate $W_{\text {exc }}^{m 2} \approx 1 / T_{1} \approx 10^{3} \mathrm{~s}^{-1}$, the populations of levels 1 and $m$ become comparable at a temperature of $T_{\text {eff }} \approx 10000 \mathrm{~K}$.

The optical density of the $1 \rightarrow 2$ transition is defined by the expression

$\tau_{12}=\sigma_{12} N_{1} D^{*} \simeq \tau_{12}^{m}\left(\frac{N_{1}}{N_{m}}\right)$

where $D^{*} \leq D$ is the size of the passive zone of the blob volume, in which diffused $\operatorname{Ly} \alpha$ radiation has the effective temperature $T_{\text {eff }}(\operatorname{Ly} \alpha)$. The maximum value of the optical density in the saturation range (Fig. 6) is

$\tau_{12}^{m}=\sigma_{12} N_{m} D^{*}$,

where

$\sigma_{12}=\frac{\lambda_{12}^{2}}{2 \pi} \frac{A_{21}}{2 \pi \Delta \nu_{\mathrm{D}}}$

is the cross-section for absorption of the UV lines, and $\Delta \nu_{\mathrm{D}}$ is the Doppler width of the UV spectral lines.

According to the observational data (see e.g. Morse et al. 1999; Johansson \& Zethson 1999; Johansson et al. 1996), gaseous blobs close to the central star produce a complex emission line spectrum consisting of surprisingly narrow lines with spectral widths simply implying an internal velocity dispersion within the blobs on the order of $20 \mathrm{~km} \mathrm{~s}^{-1}$. The relative spectral width of the UV lines

$\frac{\Delta \nu^{\text {obs }}}{\nu} \leq \frac{1}{R} \simeq 4 \times 10^{-5}$

is limited by the resolution $R \simeq 25000$ of the spectral measurements. This spectral width in the case of Doppler broadening $\Delta \nu_{\mathrm{D}}$ corresponds to the kinetic temperature $T_{\text {kin }}$ of Fe II:

$\frac{\Delta \nu_{\mathrm{D}}}{\nu_{12}}=\frac{1}{c}\left(2 \ln 2 \frac{k T_{\mathrm{kin}}}{M}\right)^{1 / 2} \leq \frac{1}{R}$

or $T_{\text {kin }} \leq 20000 \mathrm{~K}$, where $M$ is the mass of Fe. According to conditions (29) and (31), the following estimate for $\sigma_{12}$ is valid:

$\sigma_{12} \geq 7 \times 10^{-14}\left[\mathrm{~cm}^{2}\right]$.

This upper-bound estimate of $T_{\text {kin }}$ is known to be higher than the actual temperature in the passive zone, since 
only some fraction, $10^{-3}-10^{-4}$, of the particles is subject to photoionization, and naturally the deposition of energy in the form of the photoelectron kinetic energy is not very high. The kinetic energy of the Fe II ions in the dense, weakly ionized, cold HI region is probably in the range $1000-2000 \mathrm{~K}$. In that case, the Doppler broadening of the Fe II lines may be smaller and, accordingly, $\sigma_{12}$ may reach as high a value as $3 \times 10^{-13} \mathrm{~cm}^{2}$. If so, with the density $N($ Fe II $) \simeq 10^{-4} N_{0} \simeq 10^{3}-10^{4} \mathrm{~cm}^{-3}$, the very low concentration of Fe II in the initial low-lying metastable state $N_{m}$ will be sufficient for the UV transition $2 \rightarrow 1$ to have an optical density $\tau_{12}^{m} \gg 1$. Indeed, for $\tau_{12}^{m} \simeq 10^{3}-10^{4}$, the necessary concentration $N_{m}(\mathrm{Fe}$ II $) \simeq$ $3-30 \mathrm{~cm}^{-3}$, i.e., $N_{m}$ (Fe II $) \simeq 3 \times 10^{-3}-10^{-4} N($ Fe II $)$ and $D^{*} \simeq D$. This seems quite possible even at a low concentration of electrons of low kinetic energy because the energy of level $m$ is about $1 \mathrm{eV}$.

The possibility of accumulation of Fe II ions in the long-lived state 1 (see right-hand scale for $N_{1} / N_{m}$ in Fig. 6) shows, firstly, that the UV radiative transition $1 \rightarrow 2$ can be optically dense. As is shown below, this provides a key to the explanation of the abnormal ratio between the intensities of the UV Fe II lines. Secondly, this provides grounds for considering the possibility of photoionization of Fe II in the state 1 by the same Ly $\alpha$ radiation, i.e., the production of the photoions Fe III (Johansson et al. 2000) in the passive, cold region HI. This will automatically explain the appearance of the forest of narrow recombination lines in the Fe II spectrum observed.

\section{Photoionization of the long-lived states 1 of the Fe II UV transition}

The energy of the Ly $\alpha$ photons, $10.25 \mathrm{eV}$, is sufficient for the photoionization of Fe II from the long-lived states $c^{4} \mathrm{~F}_{7 / 2,9 / 2}$ with binding energies of $9.97 \mathrm{eV}$. The rate of this photoionization of the lower levels 1 by Ly $\alpha$ radiation in the blob is defined by the expression

$$
\begin{aligned}
W_{\mathrm{ph}}^{1 i} & =4 \pi \int_{I_{\mathrm{Fe}^{*}}}^{\infty} P_{\nu, T}^{\mathrm{b}} \sigma_{\mathrm{ph}}^{1 i}(\nu) \mathrm{d} \nu \\
& \simeq 4 \pi P_{\nu, T}^{\mathrm{b}}(\operatorname{Ly} \alpha) \Delta \nu_{\mathrm{Ly} \alpha} \sigma_{\mathrm{ph}}^{1 i}(\nu),
\end{aligned}
$$

where $I_{\mathrm{Fe}^{*}}$ is the ionization potential of Fe II in the states $c^{4} \mathrm{~F}_{7 / 2,9 / 2}(9.94 \mathrm{eV}), P_{\nu, T}^{\mathrm{b}}$ is the spectral intensity of Ly $\alpha$ radiation in the passive volume of the blob with the effective temperature $T_{\text {eff }}^{b}$ given by $(9), \sigma_{\mathrm{ph}}^{1 i}$ is the photoionization cross-section of Fe II from the lower states, and $\Delta \nu_{\mathrm{Ly} \alpha}$ is the spectral bandwidth of HLy $\alpha$ in the blob $\left(320 \mathrm{~cm}^{-1}\right)$. According to Nahar \& Pradhan (1994), the frequency dependence of $\sigma_{\mathrm{ph}}(\nu)$ for Fe II has a strongly non-regular character, with many narrow resonances above the ionization limit, and with $\sigma_{\mathrm{ph}}$ varying in the range $0.1-10^{2} \mathrm{Mb}$. Figure 7 shows $W_{\mathrm{ph}}^{1 i}$ as a function of $T_{\mathrm{eff}}(\mathrm{Ly} \alpha)$ for various $\sigma_{\mathrm{ph}}^{1 i}$ values: $\sigma_{\mathrm{ph}}^{1 i}=10^{-17}-10^{-19} \mathrm{~cm}^{2}(10-0.1 \mathrm{Mb})$. In case of $T_{\text {eff }}(\mathrm{Ly} \alpha) \simeq 10^{4} \mathrm{~K}$ and $\sigma_{\mathrm{ph}}^{1 i} \geq 10 \mathrm{Mb}$ this rate of photoionization can exceed the rate of radiative decay of

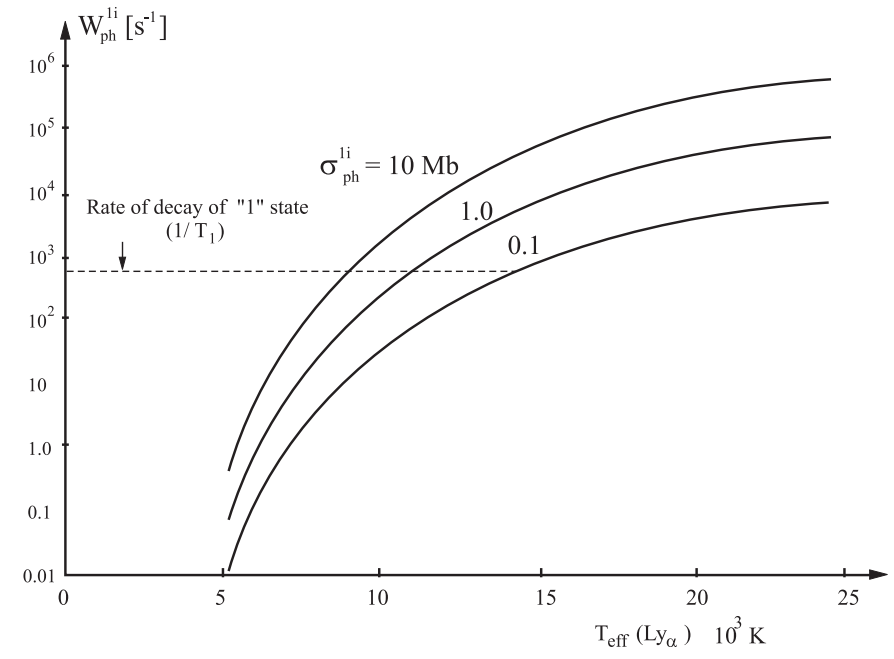

Fig. 7. Photoionization rate of $\mathrm{Fe}^{*} \mathrm{II}$ by $\mathrm{HLy} \alpha$ radiation $W_{\mathrm{ph}}^{1 i}$ in the blob as a function of the effective temperature of HLy $\alpha$ for various cross-sections of photoionization $\sigma_{\mathrm{ph}}^{1 i}$.

the low level 1, i.e. it can provide significant depletion of this level and a production of Fe III in the cold, passive HI zone of blob B.

The photoionization rate of the lower level 1 by blackbody radiation $(\mathrm{BBR})$ from the central star $\eta$ Car in the spectral window $h \Delta \nu=9.97 \mathrm{eV}-13.6 \mathrm{eV}\left(h \nu_{\mathrm{Fe}}{ }^{\mathrm{II}}=\right.$ $9.97 \mathrm{eV}$ is the ionization energy of $\mathrm{Fe}^{*}$ II in the excited state $1, h \nu_{\mathrm{c}}=13.6 \mathrm{eV}$ is the photoionization energy of $\mathrm{HI}$, which is absorbed at the short wavelength edge of the stellar radiation) is determined by the expression

$W_{\mathrm{ph}}^{1 i}(\mathrm{BBR}) \simeq \Omega \int_{\nu_{\mathrm{Fe}} \mathrm{II}^{\prime}}^{\nu_{\mathrm{c}}} P^{\mathrm{s}}\left(T_{\mathrm{eff}}^{\mathrm{s}}, \nu\right) \sigma_{\mathrm{ph}}^{1 i}(\nu) \mathrm{d} \nu$

where $\Omega$ is the dilution factor (9) of the radiation from the central star, $P^{\mathrm{s}}\left(T_{\text {eff }}^{\mathrm{s}}, \nu\right)$ is the Planck black body radiation spectral brightness of the star's photosphere with an effective temperature $T_{\mathrm{eff}}^{\mathrm{s}} \approx(20-30) \times 10^{3} \mathrm{~K}$ (Davidson \& Humphreys 1997), $\sigma_{\mathrm{ph}}^{1 i}(\nu)$ is the spectral dependence of the photo-ionization cross-section of $\mathrm{Fe}^{*} \mathrm{II}$ from the state 1 , which varies in the range $0.1-100 \mathrm{Mb}$. For a crude estimation of the order of magnitude of $W_{\mathrm{ph}}^{1 i}$ we can use the expression

$W_{\mathrm{ph}}^{1 i}(\mathrm{BBR}) \simeq \Omega P^{\mathrm{s}}\left(T_{\mathrm{eff}}^{\mathrm{s}}\right)\left(\nu_{\mathrm{c}}-\nu_{\mathrm{Fe} \mathrm{II}^{*}}\right)\left\langle\sigma_{\mathrm{ph}}^{1 \mathrm{i}}\right\rangle \Delta \nu$,

where $\left\langle\sigma_{\mathrm{ph}}^{1 i}\right\rangle$ is the effective cross-section of photoionization of $\mathrm{Fe}^{*}$ II which is similarly introduced as $\sigma_{\mathrm{ph}}$ for $\mathrm{HI}$ in (10).

The ratio of rates of these two photoionization channels is

$$
\begin{aligned}
\frac{W_{\mathrm{ph}}^{1 i}(\mathrm{Ly} \alpha)}{W_{\mathrm{ph}}^{1 i}(\mathrm{BBR})} \simeq & \left(\frac{4 \pi}{\Omega}\right) \frac{\Delta \nu(\mathrm{Ly} \alpha)}{\left(\nu_{\mathrm{c}}-\nu_{\mathrm{Fe}^{*} \mathrm{II}}\right)} \frac{\sigma_{\mathrm{ph}}^{1 i}(\mathrm{Ly} \alpha)}{\left\langle\sigma_{\mathrm{ph}}^{1 i}\right\rangle_{\Delta \nu}} \exp \\
& \times\left[\frac{h \nu}{k}\left(\frac{1}{T_{\mathrm{eff}}^{\mathrm{b}}}-\frac{1}{T_{\mathrm{eff}}^{\mathrm{s}}}\right)\right]
\end{aligned}
$$

for $h \nu \approx 10 \mathrm{eV} \gg k T_{\text {eff }}$. In the case of $\eta$ Carinae and blob B, $T_{\mathrm{eff}}^{\mathrm{s}} \approx(20-30) 10^{3} \mathrm{~K}$ (Davidson \& Humphreys $1997)$ and $T_{\mathrm{eff}}^{\mathrm{b}}(\mathrm{Ly} \alpha) \approx 10 \times 10^{3} \mathrm{~K}$. The rate of photoionization by Ly $\alpha$ in the blob is much higher $\left(10^{2}-10^{3}\right.$ times $)$ 


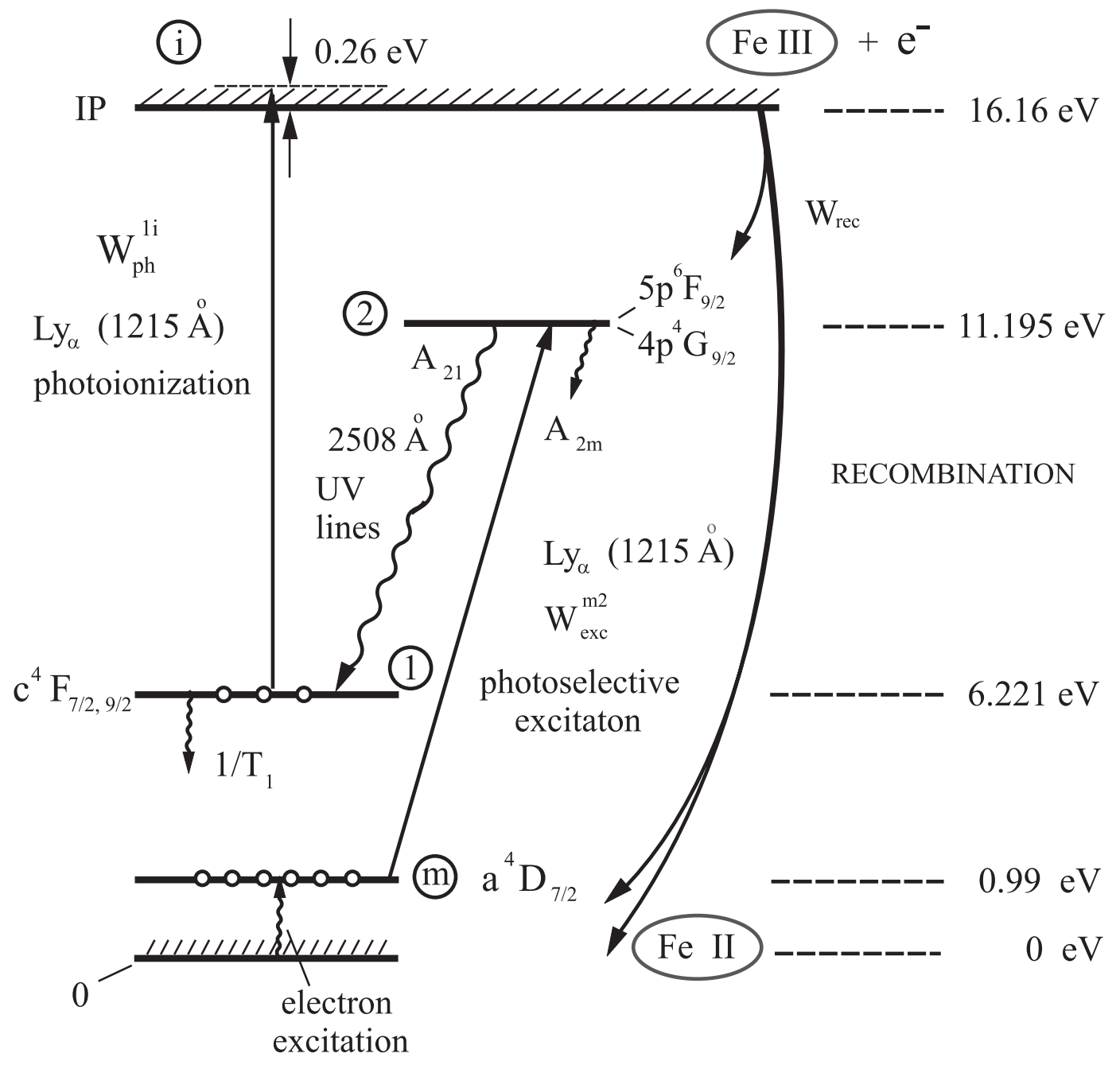

Fig. 8. Photoionization and recombination cycle: Fe II $\rightarrow$ Fe III $\rightarrow$ Fe II.

than the rate of photoionization by BBR from the central star in the range $h\left(\nu_{\mathrm{c}}-\nu_{\mathrm{Fe}^{*} \mathrm{II}}\right)$.

This implies, that the HLy $\alpha$ radiation, which is generated in the HII region and diffused into the optically thick HI region of the blob, controls the main photoprocesses involving Fe II more efficiently than the diluted radiation from the central star.

From the estimations presented above we can derive a photoionization-recombination cycle (Fig. 8) based on the accidental double resonance of the HLy $\alpha$ line with the absorption line of Fe II and the photoionization limit of Fe* II. This provides an unusually fast photoionization conversion

$$
\begin{aligned}
\mathrm{Fe}^{(m)} \mathrm{II}\left(W_{\mathrm{exc}}^{m 2}\right) & \rightarrow \mathrm{Fe}^{(2)} \mathrm{II}\left(A_{21}\right) \\
& \rightarrow \mathrm{Fe}^{(1)} \mathrm{II}\left(W_{\mathrm{ph}}^{1 i}\right) \rightarrow \mathrm{Fe} \text { III. }
\end{aligned}
$$

The slowest channels in (37) are the photoexcitation $W_{\text {exc }}^{m 2}$ and the photoionization $W_{\mathrm{ph}}^{1 i}$. The resulting rate of the photoionization conversion process $\mathrm{Fe}$ II $\rightarrow \mathrm{Fe}$ III is about $10^{3} \mathrm{~s}^{-1}$. This rate is much higher than the recombination rate $W_{\text {rec }}$. For Fe III, and for $n_{\mathrm{e}} \simeq 10^{3}-10^{4} \mathrm{~cm}^{-3}$ and $T_{\mathrm{e}} \simeq 10^{4} \mathrm{~K}, W_{\text {rec }} \simeq\left(10^{-9}-10^{-8}\right)\left[\mathrm{s}^{-1}\right]$.
However, the relatively rapid step-wise photoionization of Fe II by the trapped Ly $\alpha$ radiation cannot convert Fe II to Fe III entirely because of the low rate of collisional excitation of Fe II to the metastable $\mathrm{a}^{4} \mathrm{D}_{7 / 2}$ state (from the ground state of Fe II or by recombination of Fe III). The partial population in the ground state and other metastable states of Fe II, not excitable by Ly $\alpha$, limits the rate of the photoionization conversion Fe II $\rightarrow$ Fe III. As a result Fe II and Fe III should coexist in the blob. Observations of Fe III lines in the blob spectrum (Johansson et al. 2000) support this biphotonic mechanism of photoionization in the cold, passive zone blob B.

Let us note that the low rates of recombination for Fe II and Fe III explain the existence of low-excitation Fe II lines in the 2-months $\left(10^{7} \mathrm{~s}\right)$ period after the disappearance or weakening of the bright, high-excitation UV lines of Fe II in connection with the "spectroscopic event" (Gull, private communication), because the lowexcitation and recombination rates are determined by electron collisional excitation, but the intense UV Fe II lines are determined by the HLy $\alpha$ photo-selective excitation. 


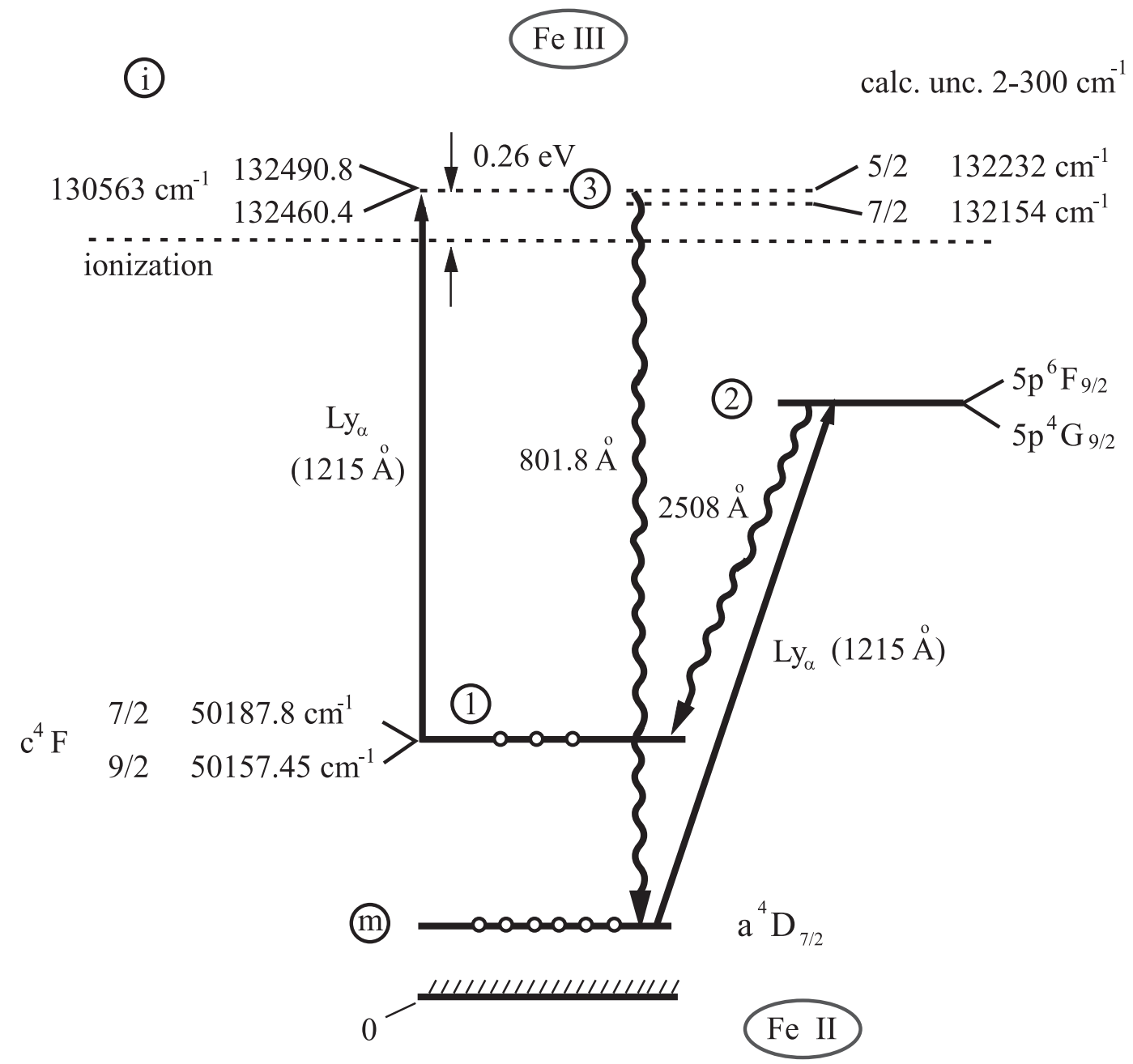

Fig. 9. Pure photonic collision-free cycle: $\mathrm{Fe}^{\mathrm{m}} \mathrm{II} \rightarrow \mathrm{Fe}$ III (above ionization) $\rightarrow \mathrm{Fe}^{m} \mathrm{II}$.

Furthermore, we should mention the possibility of a pure radiative and collision-free cycle of excitation for Fe II from levels 1 to two bound states slightly above the ionization limit with a subsequent fast radiative decay directly to the metastable level $m$, as shown in Fig. 9. This possibility is based on the existence of two energy levels of Fe II, whose predicted energies (Kurucz 1988) yield transitions that are red-shifted about $300 \mathrm{~cm}^{-1}$ relative to the HLy $\alpha$ frequency. However, the discrepancy of about $300 \mathrm{~cm}^{-1}$ is within the typical uncertainties for energy level predictions in complex spectra, such as Fe II. The present collision-free cycle can enhance the rate of $\mathrm{Fe}$ II cycling under action of Ly $\alpha$ light and the corresponding effective conversion of the Ly $\alpha$ line into the UV Fe II lines.

\section{Conclusion}

This paper presents a qualitative model of the origin of the anomalously bright UV lines of Fe II at $2507 \AA$ and $2509 \AA$ in spectra of compact gaseous condensations (blobs) in the vicinity of the star $\eta$ Car. The model is based on the idea (Johansson \& Hamann 1993) of a Bowen mechanism with photo-selective pumping of Fe II to highly-excited levels by the HLy $\alpha$ radiation within the framework of the stellar parameters of $\eta$ Car and blob B reported in Morse et al. (1999) and Davidson \& Humphreys (1997).

The simple model presented above of the active and passive regions of the blob (from the standpoint of the generation of the HLy $\alpha$ radiation) uses fairly simplified estimates, whose accuracy is of the same order of magnitude as that, to which the parameters of the blobs in the vicinity of $\eta$ Car are known today. Nevertheless, this model can be used to explain the fact that it is only the blob B that exhibits exceptionally bright UV Fe II lines. The model can also be used to explain the periodical intensity variations of Fe II emission lines (Gull, private communication) occurring with the periodical variations of $\eta$ Car (Damineli et al. 2000).

The model presented predicts a significant optical thickness for the UV Fe II transitions at $2507 \AA$ and $2509 \AA$ as well as the dependence of this optical thickness on the photoionization depletion of the excited longlived state of Fe II by the intense trapped HLy $\alpha$ radiation. These effects allow one to explain the anomalous intensity ratio between the intense UV lines and their weaker satellites, as compared with laboratory data. This effect was 
qualitatively explained by Johansson \& Letokhov (2001a), and a more detailed analysis will be presented by Klimov et al. (2001).

We are quite aware of the fact that our model does not allow for the gradual change in the intensity of the HLy $\alpha$ radiation as it diffuses from the active region into the passive region of the blob, neither does it take into account the gradual change of the electron concentration and temperature, or of the temperature of the ions HII and Fe II. The quantitative analysis of these effects will undoubtedly help to fully explain the extraordinary phenomenon of the mysterious UV Fe II lines. However, it would be advisable to make this analysis in the future, as new experimental data are obtained from the HST/STIS facility with a higher spatial and spectral resolution.

Acknowledgements. One of the authors (V. L.) is very grateful to the Swedish Natural Science Research Council (NFR) for the T. Erlander Professorship-2000, which allows him to explore the "laser-like" atomic physics with Hubble Space Telescope data in the stimulating atmosphere of Lund University. The project is also supported by the Swedish National Space Board and the Crafoord Foundation. The authors are grateful to Dr. K. Davidson, who brought to our attention the strange Fe II lines in early HST spectra of $\eta$ Car. We are also grateful to Drs. T. Gull and K. Ishibashi for providing fully reduced STIS spectra of $\eta$ Car, from which Dr. T. Zethson extracted Figure 1 for us. We also thank Dr. T. Gull for reading the submitted manuscript and giving valuable comments.

\section{References}

Adams, T. F. 1972, ApJ, 174, 439

Damineli, A., Kaufer, A., Wolf, B., et al. 2000, ApJ, 528, L101

Davidson, K., Ebbets, D., Weigelt, G., et al. 1995, ApJ, 109, 1784
Davidson, K., Ebbets, D., Johansson, S., et al. 1997, ApJ, 113, 335

Davidson, K., \& Humphreys, R. M. 1997, ARAA, 35, 1

Gull, T., Davidson, K., \& Ishibashi, K. 1999, in Eta Carinae at the millenium, ed. J. A. Morse, R. M. Humphreys, \& A. Damineli, ASP Conf. Ser., 179, 144

Hamann, F., Davidson, K., Ishibashi, K., et al. 1999, in Eta Carinae at the millenium, ed. J. A. Morse, R. M. Humphreys, \& A. Damineli, ASP Conf. Ser., 179, 116

Harrington, J. P. 1973, MNRAS, 162, 43

Hummer, D. G., \& Kunasz, P. B. 1980, ApJ, 236, 609

Johansson, S. 1978, Phys. Scr. 18, 217

Johansson, S., \& Jordan, C. 1984, MNRAS, 210, 239

Johansson, S., \& Hamann, F. 1993, Phys. Scr., T47, 157

Johansson, J., Davidson, K., Ebbets, D., et al. 1996, in Science with Hubble Telescope-II, ed. P. Benvenuti, F. D. Macehetto, \& F. J. Schreier, Space Telescope Institute, 361

Johansson, S., \& Zethson, T. 1999, in Eta Carinae at the Millenium, ed. J. A. Morse, R. M. Humphreys, \& A. Damineli, ASP Conf. Ser., 179, 171

Johansson, S., Zethson, T., Hartman, H., et al. 2000, A\&A, 361, 977

Johansson, S., \& Letokhov, V. S. 2001a, in Eta Carinae and other Mysterious Stars, ed. T. R. Gull, S. Johansson, \& K. Davidson, ASP Conf. Ser., 242, 309

Klimov, V., Johansson, S., \& Letokhov, V. 2001, A\&A, submitted

Kurucz, R. L. 1988, Trans. IAOXXB, ed. M. NcNally (Kluwer, Dordrecht), 168

Mihalas, D. 1978, Stellar Atmospheres (W.H. Freeman and Company, N.Y.)

Morse, J. A., Humphreys, R. M., Damineli, A. (ed.) 1999, Eta Carinae at the Millenium, ASP Conf. Ser., 179

Nahar, S. N., \& Pradhan, A. K. 1994, J. Phys. B. At. Mol. Phys. 27, 429

Shevelko, V. P., \& Vainstein, L. A. 1993, Atom physics and hot plasmas. (IOP, Bristol)

Weigelt, G., \& Ebersberger, J. 1986, A\&A, 163, L5 